\title{
The Levels of Wnt5a and Its Receptors Frizzled5 and Frizzled2 as Immunohistochemical Biomarkers of Severity of Psoriasis
}

\author{
Xiaoying Ning \\ Dingwei Zhang \\ Yuan Wang \\ Jia Huo \\ Ying Huang \\ Ying Guo \\ Zhengxiao Li (D) \\ Yanfei Zhang
}

Department of Dermatology, The Second Affiliated Hospital of Xi'an Jiaotong University, Xian, Shaanxi Province,

People's Republic of China
Correspondence: Zhengxiao Li; Yanfei Zhang

Department of Dermatology, The Second Affiliated Hospital of Xi'an Jiaotong University, 157 Xi Wu Road, Xian,

Shaanxi Province, 710004 , People's

Republic of China

Tel/Fax +86-29-8767930l

Email lizhengxiao1979@।63.com;

zhangyanfei723@163.com
Purpose: Psoriasis is a systemic, chronic and inflammatory condition. The exact pathogenesis is unclear. The abnormal expression of Wnt5a pathway in psoriasis vulgaris has been confirmed. Whether it is related to the severity of psoriasis is unclear.

Methods: Thirty-eight skin lesions from psoriasis vulgaris patients and 22 healthy adult skin tissues were taken. The semi-quantitative immunohistochemistry score of Wnt5a, Frizzled5 and Frizzled 2 was evaluated under a microscope by two independent dermatologists. Psoriasis area and severity index (PASI) score system was used to evaluate the disease severity.

Results: The average PASI score of the patients was $16.25 \pm 7.8$, and the average duration of disease was $19.6 \pm 10.4$ months. Wnt5a, Frizzled5 and Frizzled2 were highly expressed in psoriasis lesions. The semi-quantitative immunohistochemistry scores of Wnt5a, Frizzled5 and Frizzled 2 were positively correlated with PASI scores $(r=0.71, r=0.46, r=0.65$, respectively, all $P$-value $<0.01)$, but not correlated with duration of disease $(r=0.11, r=$ $0.17, \mathrm{r}=0.29$, respectively, all $P$-value $>0.05)$. There were significant positive correlations between Wnt5a and Frizzled5 $(\mathrm{r}=0.57, P$-value $<0.01)$, as well as Wnt5a and Frizzled2 $(\mathrm{r}=$ $0.59, P$-value $<0.01)$.

Conclusion: Wnt5a and its receptors play an important role in pathogenesis of psoriasis vulgaris and are positively correlated with the severity of psoriasis, and may be one of the immunohistochemical predictors of the severity of the disease.

Keywords: psoriasis vulgaris, Wnt5a, PASI, Frizzled5, Frizzled2

\section{Introduction}

Psoriasis is a chronic and systemic inflammatory condition with a prevalence is approximately 2-3\%, and it may affect more than 125 million individuals worldwide. ${ }^{1,2}$ Its related mortality, economic burden and morbidity are high, and impairing the quality-of-life of psoriasis patients markedly. Excessive proliferation of keratinocytes, and infiltration of immune cells into the skin is involved in psoriasis, but the exact pathogenesis is unclear. ${ }^{3}$ The crucial role of Wnt5a in psoriasis had been investigated. ${ }^{4-6}$

The Wnts are a large family with cysteine-rich secreted glycoproteins, which play an important role in the development and physiological processes of many tissues. ${ }^{7}$ As a key factor in the non-classical Wnt pathways, Wnt5a regulates the inflammatory response and various functions of the cells by activating a cascade signaling pathways, including cell proliferation, differentiation, apoptosis, survival, and cell polarity. ${ }^{8}$ Studies have revealed that Wnt5a gene was up-regulated in psoriatic lesions. ${ }^{4-6}$ 
Wnt5a signaling pathway can regulate downstream factors by binding related receptors, such as Frizzled5 and Frizzled2, and then to regulate cell biological function by activating $\beta$ catenin and protein kinase $\mathrm{C}$ (PKC). Wnt5a signaling pathway can regulate the proliferation, apoptosis and migration of keratinocytes. ${ }^{9}$ A recent analysis of gene pathogenicity found that Wnt5a gene is one of the five key genes and may be a biomarker for the diagnosis and treatment of psoriasis. ${ }^{10}$ It was also found in our previous studies that Wnt5a and its receptors were highly expressed in psoriatic lesions. ${ }^{11}$

So we speculate that Wnt5a pathway is not only associated with psoriasis recurrence but also with psoriasis severity. The present study was planned to evaluate the correlation between Wnt5a, Frizzled5, Frizzled2 and the severity of the psoriasis.

\section{Materials and Methods}

\section{Samples}

Patients with psoriasis vulgaris with typical clinical manifestations and confirmed by histopathology were selected as research objects. Healthy adults with normal skin as the control group. All selected patients and healthy adults had no history of drug intake (such as glucocorticoids, retinoic acid, and immunosuppressive drugs) in the past 2 months, and used no any topical drugs in the past 2 weeks. There were no history of hypertension, diabetes, obesity and other chronic diseases when patients and healthy adults were included. All participants completed the written informed consent. All biopsies were done under local anesthesia. Evaluation of Psoriasis Area and Severity Index (PASI) scores was done by dermatologist. All experimental protocols were approved by the Institutional Review Board of the Xi'an Jiaotong University, and performed according to guidelines governing ethics care in China. This study was performed in accordance with the rules laid down in the Declaration of Helsinki.

\section{Immunohistochemistry}

All tissues were embedded in paraffin. At the temperature at $4^{\circ} \mathrm{C}$, the paraffin sections were incubated with the primary anti-Wnt5a (Abcam ab110073, Cambridge, UK, final dilution 1:250), anti-Frizzled-2 (Abcam ab109094, Cambridge, UK, final dilution 1:300), and anti-Frizzled-5 (Abcam ab75234, Cambridge, UK, final dilution 1:250) antibody overnight. Then, a biocatalyst streptavidin-horseradish peroxidase-conjugated as secondary antibody (ZSGB-BIO, Beijing, China) were used, at last with hematoxylin counterstaining and 3'-diaminobenzidine (ZSGB-BIO, Beijing,
China). In negative control, phosphate buffered saline (PBS) was used to replace the primary antibody.

\section{Semi-Quantitative Analysis of Immunohistochemistry Results}

NanoZoomer Digital Pathology (Hamamatsu, Japan) was used to examine the sections. At high-power fields (200× magnifications), randomly selected five images were captured by a DP 70 CCD camera (Olympus Corp., Tokyo, Japan) for quantitative analysis. Semi-quantitative analysis of immunohistochemical results: the sample was scored with a percentage of positive cells (stain was cytoplasmic or nuclear) and a staining intensity score. ${ }^{12}$ The staining intensity score was 0 for unstained, 1 for pale yellow, 2 for brown yellow, and 3 for brown. According to the percentage of the number of positive cells, the score was divided as: 0 points for $<5 \%, 1$ point for $5 \%$ to $25 \%, 2$ points for $26 \%$ to $50 \%$, and 3 points for $>50 \%$. Multiply the degree of staining and the percentage of stained cells in each section to get the final score: 0 to 1 is considered negative, and $\geq 2$ is considered positive.

\section{Statistical Analysis}

All data were processed with SPSS19.0 statistical analysis software (SPSS Inc., Chicago, IL, USA). Unpaired $T$ test, Chi-square test was used for statistical analysis of the results. Correlation analysis was performed by the Spearman two-variable correlation analysis method. $P<0.05$ was defined as statistically differences.

\section{Results}

\section{The Characteristics of the Participants}

As shown in Table 1, 38 lesions from 38 patients with psoriasis vulgaris were enrolled (20 males and 18 females, age range 19-71 years, mean age was $50.2 \pm 13.1$ years). There were 16 lesions located at the trunk, 12 at the upper limb and 10 at the lower limb. The average PASI score was $16.25 \pm 7.8$, and the average duration of disease was $19.6 \pm 10.4$ months. Normal skin tissue of 22 healthy adults (11 males and 11 females, age range 21-68 years,

Table I Clinical Data of the Study Participants

\begin{tabular}{|l|l|}
\hline Clinical Data & Patients \\
\hline Number of patients & 38 \\
Age (years), mean $\pm S D$ & $50.2 \pm 13.1$ \\
PASI score, mean $\pm S D$ & $16.25 \pm 7.8$ \\
Duration of psoriasis (months), mean $\pm S D$ & $19.6 \pm 10.4$ \\
\hline
\end{tabular}

Abbreviations: PASI, psoriasis area and severity index; SD, standard deviation. 
Table 2 The Expression of Wnt5a, Frizzled5 and Frizzled2 in Psoriasis

\begin{tabular}{|l|l|l|l|l|l|l|l|}
\hline \multirow{2}{*}{ Groups } & \multirow{2}{*}{ N } & \multicolumn{2}{|c|}{ Wnt5a } & \multicolumn{2}{c|}{ Frizzled5 } & \multicolumn{2}{c|}{ Frizzled2 } \\
\cline { 3 - 9 } & & - & + & - & + & - & + \\
\hline Psoriasis lesions & 38 & $8(21.1 \%)$ & $30(78.9 \%)$ & $10(26.3 \%)$ & $28(73.7 \%)$ & $7(18.4 \%)$ & $31(81.6)$ \\
Healthy controls & 22 & $18(81.8 \%)$ & $4(18.2 \%)$ & $17(77.3 \%)$ & $5(22.7 \%)$ & $15(68.2 \%)$ & $7(31.8 \%)$ \\
\hline
\end{tabular}

mean age was $48.7 \pm 14.9$ years) as the control. There were no significant differences in gender and age between the two groups $(P$-value $>0.05)$.

\section{Immunohistochemical Analysis of Wnt5a, Frizzled5, and Frizzled2 in Psoriasis}

As shown in Table 2 and Figure $1 \mathrm{~A}$ and B. Thirty specimens of psoriasis had increased Wnt5a staining, not only in all the epidermal layers but also in inflammatory and endothelial cells in the dermis. In normal skin, 18 cases had no increased in Wnt5a staining, Wnt5a proteins normally staining in the basal layer, and 4 cases had increased Wnt5a staining (in addition to the basal layer, there was a small amount positive cells in the spinous layer). The expression rate of Wnt5a in psoriasis was significantly higher than it in normal skin, and the difference was statistically significant $(\chi 2=20.95, P$-value $<0.001)$.

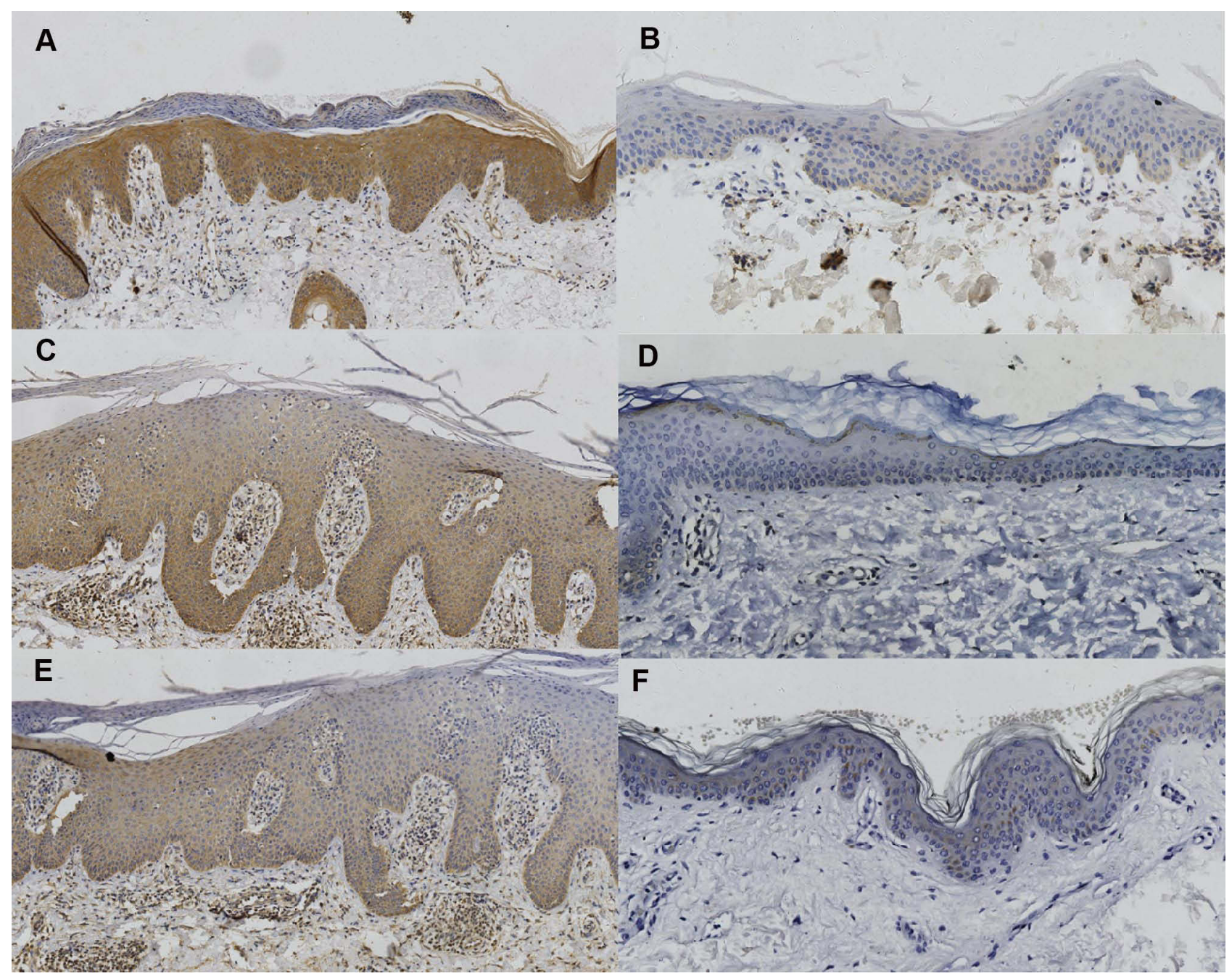

Figure I Skin sections immunostaining stained. (A) Wnt5a in lesions; (B) Wnt5a in normal skin; (C) Frizzled5 in lesions; (D) Frizzled5 in normal skin; (E) Frizzled2 in lesions; (F) Frizzled2 in normal skin. (A, C, E with $\times 100$ magnification, B, D, F with $\times 200$ magnification). 
As shown in Table 2 and Figure $1 \mathrm{C}$ and D. There were 28 cases with psoriasis with increased of Frizzled5 staining in all the epidermal layers, inflammatory and endothelial cells in the dermis. In normal skin, 17 cases had normal of Frizzled5 staining (in the granular layer), and 5 cases had increased staining (there was a small amount positive cells in the spinous layer and the basal layer). The expression of Frizzled5 in psoriatic lesions was significantly higher than it in normal skin, and the difference was statistically significant $(\chi 2=12.63, P$-value $<0.001)$.

As shown in Table 2 and Figure 1E and F, 31 psoriasis cases $(81.58 \%)$ had increased Frizzled2 staining in all the epidermal layers, inflammatory and endothelial cells in dermis. In normal skin tissues, 7 cases had slight increased staining (besides the basal layer, there was increased staining in the upper basal layer). The expression of Frizzled2 in psoriatic tissue was significantly higher than it in normal skin, and the difference was statistically significant $(\chi 2=12.79, P$-value $<$ $0.001)$.

\section{Correlation Between the Expression Levels of Wnt5a and Frizzled5, Frizzled2 in Psoriasis}

Spearman two-variable correlation coefficient analysis showed that significant positive correlations between Wnt5a and Frizzled5 ( $\mathrm{r}=0.57, P$-value $<0.01)$, as well as Wnt5a and Frizzled2 $(r=0.59, P$-value $<0.01)$ in psoriasis.

\section{Correlation Between Semi-Quantitative} Scores of Wnt5a, Frizzled5 and Frizzled2 with PASI Score and Duration of Disease

Semi-quantitative score of Wnt5a in psoriasis vulgaris lesions was positively correlated with PASI score $(\mathrm{r}=0.71, P$-value $<$ $0.01)$, but not correlation with the duration of disease $(\mathrm{r}=$ $0.11, P$-value $>0.05$ ) (Figure 2A and B). There was a positive correlation between the semi-quantitative score of Frizzled5 and the PASI score $(\mathrm{r}=0.46, P$-value $<0.01)$, and there was no correlation between the semi-quantitative score of Frizzled5 and the duration of disease $(\mathrm{r}=0.17, P$-value $>$ 0.05 ) (Figure $2 \mathrm{C}$ and D). Semi-quantitative score of Frizzled2 was positively correlated with PASI score $(\mathrm{r}=0.65, P$-value $<$ $0.01)$, but not correlated with the duration of disease $(\mathrm{r}=0.29$, $P$-value $>0.05$ ) (Figure 2E and F).

\section{Discussion}

In psoriatic lesions, Wnt5a and the receptors Frizzled2 and Frizzled5 were not only expressed in the entire epidermis but also strongly positive expressed in superficial dermal inflammatory cells and vascular endothelial cells, indicating that the Wnt5a pathways may be involved in the regulation of inflammatory response process, but its specific role in psoriasis is currently unknown.

Infiltration of inflammatory cells in the epidermis and dermis in psoriatic lesions is a crucial factor in the pathogenesis of psoriasis. Numerous factors have been found to
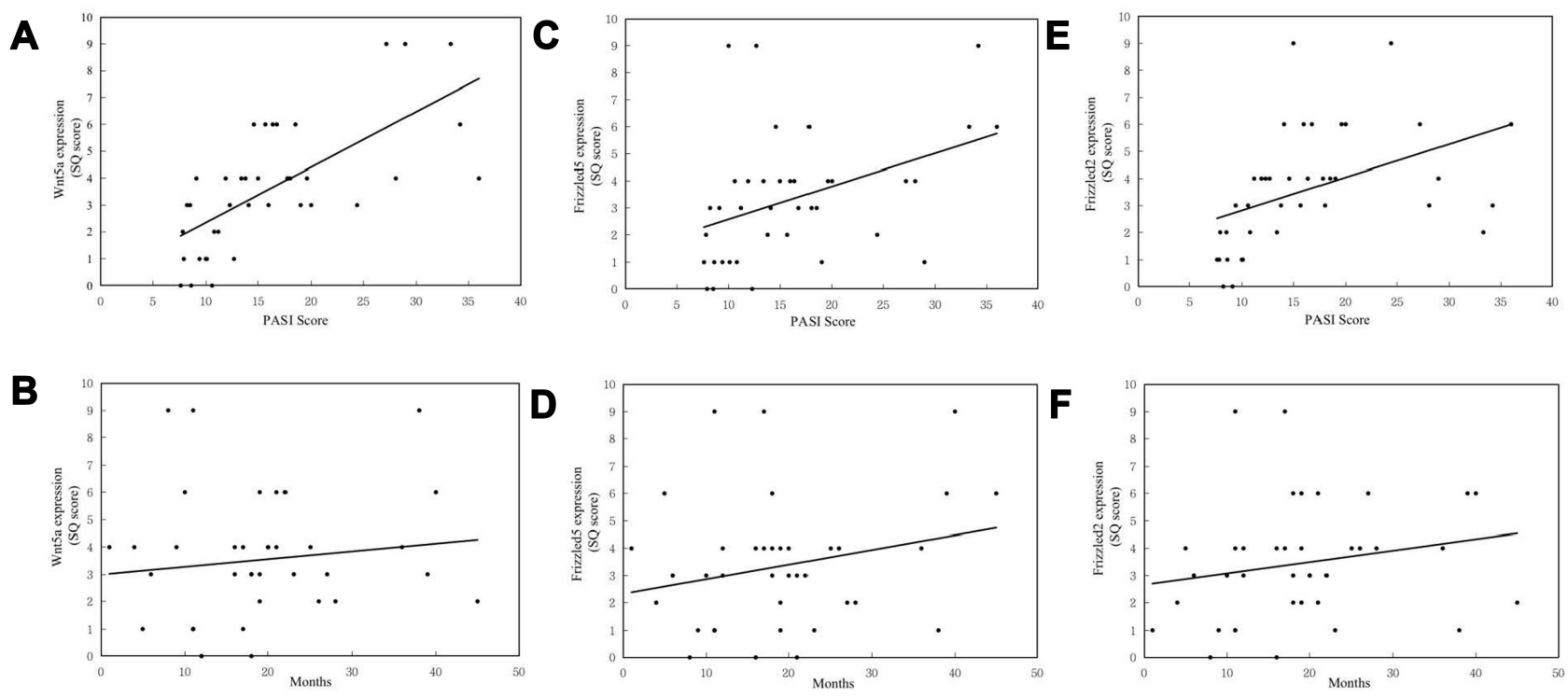

Figure 2 (A) The semi-quantitative score of Wnt5a in lesions was positively correlated with PASI score; (B) but not with duration of disease; (C) the semi-quantitative score of Frizzled5 was positively correlated with PASI score; (D) but not with duration of disease; (E) the semi-quantitative score of Frizzled2 was positively correlated with PASI score; (F) but not with duration of disease. 
participate in the pathogenesis of psoriasis vulgaris in mouse models. ${ }^{13}$ The Wnt5a pathway plays an important role in the pathogenesis of various inflammatory diseases. ${ }^{14}$ After binding to the corresponding receptors, Wnt5a activate the downstream molecular pathways, and then affects the progress of the diseases. Interventions targeting Wnt5a or its receptors and downstream factors may be one of the methods for treating inflammatory diseases. ${ }^{15} \mathrm{Wnt} 5 \mathrm{a}$ and receptors are expressed in epithelial cells, lymphocytes, endothelial cells and smooth muscle cells. ${ }^{16}$ In HaCaT cells, after treatment with Wnt5a, the expression levels of inflammatory markers, including IFN$\gamma$, IL- 8 and IL-17A were unregulated. ${ }^{17}$ IL-1 $\alpha$, TNF- $\alpha$, IFN- $\gamma$, and TGF- $\alpha$ can induce the expression of Wnt5a. ${ }^{5}$ All these inflammatory markers play critical roles in the pathogenesis and maintenance of psoriasis. ${ }^{3}$ Wnt5a also participates in the early emergency response of psoriasis to mechanical stress. ${ }^{18}$ Differential methylations of Wnt5a and Frizzled2 were found in psoriasis. ${ }^{19}$ Nuclear factors of activated T-cells, cytoplasmic1 (NFATc1), a downstream gene of Wnt5a, can induce psoriasiform dermatitis. ${ }^{20}$ Wnt5a-activated signalling pathways mediate interactions among keratinocytes, immune cells and inflammatory factors, and play an important role in the development of psoriasis and psoriatic arthritis (PsA). ${ }^{21}$ Therefore, the Wnt5a pathway may be a key factor in the inflammatory cytokines loop and enables the occurrence of cytokine storm of psoriasis.

Frizzled 2 activates the classical $\beta$-catenin and the independent $\beta$-catenin pathway by regulating the calcium ion pathway. ${ }^{22}$ Wnt5a/Frizzled2 can activate small G protein, activated small $G$ protein can activate Phospholipase C (PLC), PLC induces endoplasmic reticulum to release calcium ions, which further activate calcineurin or PKC pathway. ${ }^{22}$ This pathway is also used to treat psoriasis, eg, calcipotriol (a vitamin D3 derivative drug) inhibits psoriatic keratinocyte proliferation through PKC and PLC. Tacrolimus and cyclosporin may also inhibit the immune response of psoriasis by inhibiting phosphatase. Wnt5a/Frizzled5 pathway can induce the synthesis of TNF- $\alpha$ and IL-1, both TNF- $\alpha$ and IL-1 are involved in the pathogenesis of psoriasis. ${ }^{23}$ Wnt5a/Frizzled5 is highly expressed in rheumatoid arthritis. Fibroblasts isolated from rheumatoid joints can synthesize IL-15, IL-8 and IL-6, and then these cytokines act on the Wnt5a/Frizzled5 signaling pathway. ${ }^{24}$ In endothelial cells, Wnt5a induces Nuclear factor of activated T-cells (NFAT) transcription by binding to Frizzled5, and then induces T-cells to secrete IL-2, both NFAT and IL-2 are strongly expressed in psoriasis vulgaris. ${ }^{25}$ In our current research, Wnt5a was positively correlated with the expression of Frizzled2 and Frizzled5 in psoriasis lesions. Therefore, Wnt5a may be bind to Frizzled2 and Frizzled5, to activate related signaling pathways, and then regulate downstream factors to participate in the pathogenesis of psoriasis.

The correlation analysis found that the expression of Wnt5a, Frizzled2 and Frizzled5 were positively correlated with the severity of psoriasis vulgaris (PASI score), but not to the duration of the disease. The degree of erythema, scale and cell infiltration are important indicators for clinical evaluation of the severity of psoriasis. The Wnt and Wnt5a pathways may be as potential therapeutic targets of inflammatory disorders and skin cancer. ${ }^{26,27}$ Thus, with the study of the Wnt5a signaling pathway, it as a target for psoriasis treatment will become a reality in the future. This study also has some limitations, such as the expression of Wnt5a and its receptors Frizzled5 and Frizzled2 in skin lesions after treatment were not detected. The number of patients in this study is too small, and it needs to be further expanded, and the animal experiments need to be carried out in the future.

\section{Conclusion}

In summary, our results further strengthen the linkage between psoriasis and the Wnt5a signaling pathway. Wnt5a, Frizzled 2 and Frizzled5 could be used as immunohistochemical biomarkers of severity of psoriasis.

\section{Funding}

This work was supported by National Natural Science Foundation of China (Number: 81703129 and 81773327).

\section{Disclosure}

The authors declare no conflicts of interest in this work.

\section{References}

1. Crow JM. Psoriasis uncovered. Nature. 2012;492(7429):S50-S51. doi:10.1038/492S50a

2. WHO. Global Report on Psoriasis. Geneva: World Health Organization; 2016. Available from: https://apps.who.int/iris/handle/ 10665/204417. Accessed April 29, 2021.

3. Boehncke WH, Schön MP. Psoriasis. Lancet. 2015;386(9997): 983-994. doi:10.1016/S0140-6736(14)61909-7

4. Romanowska M, Evans A, Kellock D, et al. Wnt5a exhibits layer-specific expression in adult skin, is upregulated in psoriasis, and synergizes with type 1 interferon. PLoS One. 2009;4(4):e5354. doi:10.1371/journal.pone. 0005354

5. Gudjonsson JE, Johnston A, Stoll SW, et al. Evidence for altered Wnt signaling in psoriatic skin. J Invest Dermatol. 2010;130(7):1849-1859. doi:10.1038/jid.2010.67 
6. Reischl J, Schwenke S, Beekman JM, Mrowietz U, Stürzebecher S, Heubach JF. Increased expression of Wnt5a in psoriatic plaques. J Invest Dermatol. 2007;127(1):163-169. doi:10.1038/sj.jid.5700488

7. Ng LF, Kaur P, Bunnag N, et al. WNT signaling in disease. Cells. 2019;8(8):826. doi:10.3390/cells8080826

8. Kumawat K, Gosens R. WNT-5A: signaling and functions in health and disease. Cell Mol Life Sci. 2016;73(3):567-587. doi:10.1007/ s00018-015-2076-y

9. Tetreault MP, Weinblatt D, Shaverdashvili K, Yang Y, Katz JP. KLF4 transcriptionally activates non-canonical WNT5A to control epithelial stratification. Sci Rep. 2016;6:26130. doi:10.1038/srep26130

10. Dou J, Zhang L, Xie X, et al. Integrative analyses reveal biological pathways and key genes in psoriasis. Br J Dermatol. 2017;177 (5):1349-1357. doi:10.1111/bjd.15682

11. Zhang Y, Tu C, Zhang D, et al. Wnt/ $\beta$-Catenin and Wnt5a/Ca pathways regulate proliferation and apoptosis of keratinocytes in psoriasis lesions. Cell Physiol Biochem. 2015;36(5):1890-1902. doi:10.1159/ 000430158

12. Pavlakis K, Messini I, Athanassiadou S, et al. Endocervical glandular lesions: a diagnostic approach combining a semi-quantitative scoring method to the expression of CEA, MIB-1 and p16. Gynecol Oncol. 2006;103(3):971-976. doi:10.1016/j.ygyno.2006.06.012

13. Nakajima K, Sano S. Mouse models of psoriasis and their relevance. $J$ Dermatol. 2018;45(3):252-263. doi:10.1111/1346-8138.14112

14. Pashirzad M, Shafiee M, Rahmani F, et al. Role of Wnt5a in the pathogenesis of inflammatory diseases. J Cell Physiol. 2017;232 (7):1611-1616. doi:10.1002/jcp. 25687

15. Zhang CJ, Zhu N, Liu Z, et al. Wnt5a/Ror2 pathway contributes to the regulation of cholesterol homeostasis and inflammatory response in atherosclerosis. Biochim Biophys Acta Mol Cell Biol Lipids. 2020;1865(2):158547. doi:10.1016/j.bbalip.2019.158547

16. Arderiu G, Espinosa S, Peña E, Aledo R, Badimon L. Monocytesecreted Wnt5a interacts with FZD5 in microvascular endothelial cells and induces angiogenesis through tissue factor signaling. J Mol Cell Biol. 2014;6(5):380-393. doi:10.1093/jmcb/mju036

17. Wang W, Yu X, Wu C, Jin H. Differential effects of Wnt5a on the proliferation, differentiation and inflammatory response of keratinocytes. Mol Med Rep. 2018;17(3):4043-4048. doi:10.3892/ mmr.2017.8358
18. Tönük ŞB, Yorgancıŏlu ZR. Biomechanical factors in psoriatic disease: defective repair exertion as a potential cause. Hypothesis presentation and literature review. ACR Open Rheumatol. 2019;1 (7):452-461. doi:10.1002/acr2.11056

19. Verma D, Ekman AK, Bivik Eding C, Enerbäck C. Genome-wide DNA methylation profiling identifies differential methylation in uninvolved psoriatic epidermis. J Invest Dermatol. 2018;138(5):10 88-1093. doi:10.1016/j.jid.2017.11.036

20. Alrefai H, Muhammad K, Rudolf R, et al. NFATc1 supports imiquimod-induced skin inflammation by suppressing IL-10 synthesis in B cells. Nat Commun. 2016;7:11724. doi:10.1038/ncomms11 724

21. Tian F, Mauro TM, Li Z. The pathological role of Wnt5a in psoriasis and psoriatic arthritis. J Cell Mol Med. 2019;23(9):5876-5883. doi: $10.1111 /$ jcmm.14531

22. Sato A, Yamamoto H, Sakane H, Koyama H, Kikuchi A. Wnt5a regulates distinct signalling pathways by binding to Frizzled2. EMBO J. 2010;29(1):41-54. doi:10.1038/emboj.2009.322

23. Li B, Zhong L, Yang X, Andersson T, Huang M, Tang SJ. WNT5A signaling contributes to $\mathrm{A} \beta$-induced neuroinflammation and neurotoxicity. PLoS One. 2011;6(8):e22920. doi:10.1371/journal.pone.00 22920

24. Sen M, Chamorro M, Reifert J, Corr M, Carson DA. Blockade of Wnt-5A/frizzled5 signaling inhibits rheumatoid synoviocyte activation. Arthritis Rheum. 2001;44(4):772-781. doi:10.1002/15290131(200104)44:4<772::AID-ANR133>3.0.CO;2-L

25. Murphy LL, Hughes CC. Endothelial cells stimulate T cell NFAT nuclear translocation in the presence of cyclosporin A: involvement of the wnt/glycogen synthase kinase-3 beta pathway. J Immunol. 2002;169(7):3717-3725. doi:10.4049/jimmunol.169.7.3717

26. Nusse R, Clevers H. Wnt/ $\beta$-catenin signaling, disease, and emerging therapeutic modalities. Cell. 2017;169(6):985-999. doi:10.1016/j. cell.2017.05.016

27. Jenei V, Sherwood V, Howlin J, et al. A t-butyloxycarbonyl-modified Wnt5a-derived hexapeptide functions as a potent antagonist of Wnt5a-dependent melanoma cell invasion. Proc Natl Acad Sci USA. 2009;106(46):19473-19478. doi:10.1073/pnas.0909409106

\section{Publish your work in this journal}

Clinical, Cosmetic and Investigational Dermatology is an international, peer-reviewed, open access, online journal that focuses on the latest clinical and experimental research in all aspects of skin disease and cosmetic interventions. This journal is indexed on CAS.
The manuscript management system is completely online and includes a very quick and fair peer-review system, which is all easy to use. Visit http://www.dovepress.com/testimonials.php to read real quotes from published authors. 